\title{
Vibration isolation support system for a truncated icosahedral gravitational wave antenna
}

\author{
W. F. Velloso, Jr. ${ }^{\text {a) }}$ \\ Instituto Astronômico e Geofísico-USP. Av. Miguel Stefano 4200, 04301-São Paulo, SP, Brazil \\ J. L. Melo and O. D. Aguiar \\ Instituto Nacional de Pesquisas Espaciais-INPE. Av. dos Astronautas 1758-12227-010-São José dos \\ Campos, SP, Brazil
}

(Received 12 November 1999; accepted for publication 8 February 2000)

\begin{abstract}
We designed a mechanical isolation system for an icosahedral resonant gravitational wave detector we plan to construct in Brazil. We have used the NASTRAN finite element software to perform the numerical analysis. Our results show that the designed system could allow a damping factor better than $-200 \mathrm{~dB}$ in the spectral range of interest, which is adequate to the sensibility level we want for the antenna. (C) 2000 American Institute of Physics. [S0034-6748(00)00806-6]
\end{abstract}

\section{INTRODUCTION}

Although a direct detection of gravitational radiation has not been achieved yet, the existence of such radiation is already widely accepted. Several binary systems of compact stars were discovered (the first of them in 1974) for which the orbital period is decreasing. The amount of decreasing observed coincides, with great precision, with the one predicted by general relativity if gravitational radiation is been emitted. Because of this indirect detection Taylor and Hulse ${ }^{1}$ won the Physics Nobel Prize in 1993.

Nowadays, there are two methods more frequently used in the different laboratories around the world for the detection of gravitational waves: the monitoring of the distance between two or more points in space, or the measurement of the mechanical oscillations induced in the resonant modes of a material body. The first of these detection methods can be applied with the aid of a "laser" interferometer or a "Doppler' radar, and the second one with a solid body (in this case, the body's mechanical vibrations are transformed into electrical signals by electromechanical transducers, and these signals are amplified and taped on magnetic tapes for posterior analysis).

Resonant-mass detectors originated from the pioneering work of Weber. They contain a resonant mass, usually a cylindrical bar, carefully suspended in vacuum so that it is free to vibrate in the presence of a gravitational wave. Among these detectors the most sensitive now operating are cooled down to liquid helium temperatures $(2-4.2 \mathrm{~K}$ or below). They belong to the so called second and third generations of resonant-mass detectors, while those belong to the first generation operated at room temperature.

The most sensitive operating detectors [located at Louisiana (USA), Geneve (Switzerland), Rome and Padua (Italy) and Perth (Australia)] are sensitive to gravitational waves produced in a supernova explosion occurring in our galaxy. This is not very promising, though, because the rate of oc-

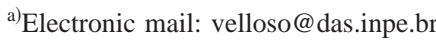

currence of such events is, optimistically, one in ten years. In order to increase this rate to one event per month it is necessary to monitor a greater volume of stars in space, and to attain this goal two main strategies have been devised. The first of them is adopted by the groups that use laser interferometry: they are willing to build interferometers with arms 100 times longer than those of the biggest interferometers so far constructed. The other strategy, adopted by groups that use resonant-mass detectors, aims to increase the sensitivity of the present detectors in five orders of magnitude in energy.

To this end, some groups have already finished the construction of third generation detectors, like NAUTILUS and AURIGA, that use bars cooled down to ultracryogenic temperatures (around $40 \mathrm{mK}$ ). Also, a fourth generation of detectors have been planned, for which the resonant mass would have a spherical geometry, for example buckyballs, and would be cooled down to ultracryogenic temperatures (EINSTEIN, TIGA, ELSA, and GRAIL). ${ }^{2}$ These detectors, which use bodies with much greater masses than the preceding ones and a more favorable geometry, are expected to be as sensitive as the giant interferometers, with the advantage of costing 15 times less. In theory such systems are omnidirectional, which means that they have the same sensitivity in all directions in space, and would be able to determine the direction and the polarization of gravitational waves emitted by supernovas or inspiralling binaries located at distances of around some hundred millions light years. To achieve the necessary sensibility the buckyball would be made with a material of high mechanical quality (high $Q_{\text {mec }}$ ), kept in vacuum, cooled down to less than $0.1 \mathrm{~K}$, and protected from electromagnetic noise and from excessive cosmic ray flux and from the mechanical vibrations of the laboratory.

The Brazilian Graviton Project plans to construct the following detectors:

(1) the Mario SCHENBERG detector ${ }^{3}$-a 0,6 m diameter spheroidal gravitational wave detector of reduced size, which will be a prototype for larger size spheroidal detectors;

(2) the NEWTON detector - a spheroidal gravitational 


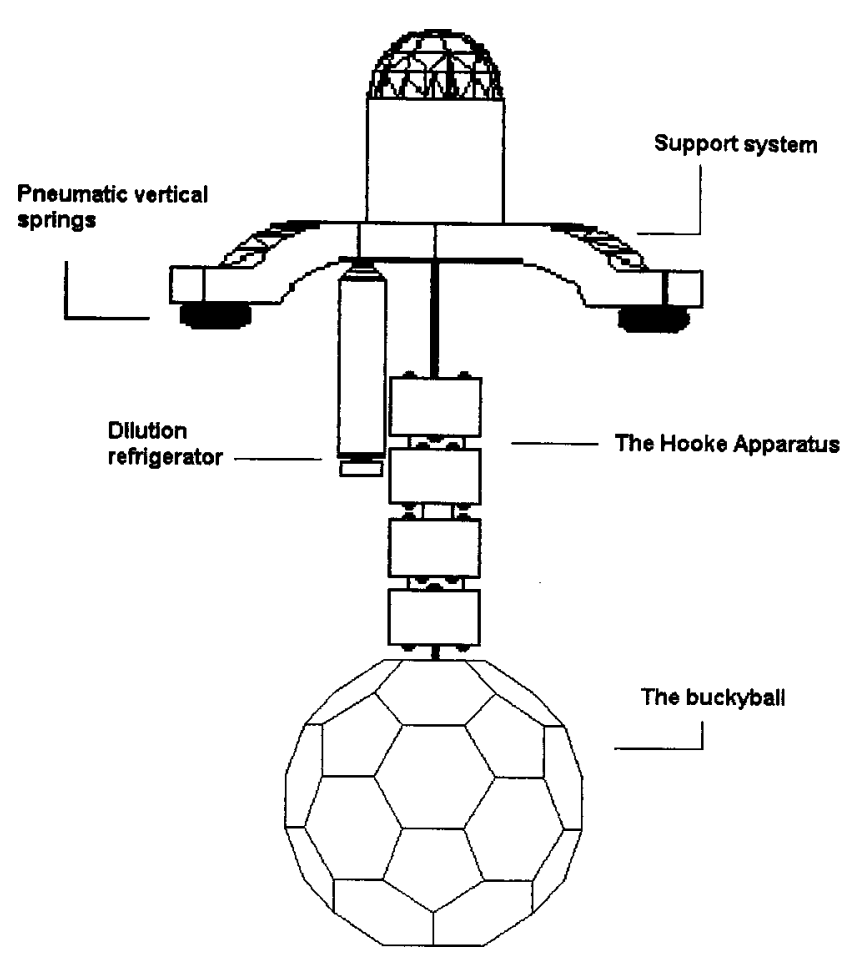

FIG. 1. The NEWTON antenna schematics.

wave detector of medium size ( $1 \mathrm{~m}$ diameter), which will work in coincidence detection with the SCHENBERG detector and also will cover a lower frequency bandwidth;

(3) the EINSTEIN detector-a $3 \mathrm{~m}$ diameter high sensitivity spheroidal gravitational wave detector, which will complete the frequency bandwidth.

The vibration isolation system for the antenna suspension, as well as for assuring a good cooling down without reintroducing vibrations in the detector, is an aspect of crucial importance in any gravitational detector project.

In this article we describe the vibration isolation system we have designed for the NEWTON medium size detector.
The basic idea used in the system design was to construct a mechanical multistage low pass filter capable of filtering all the mechanical vibration noises. The dynamical behavior of the system-which we named HOOKE Apparatus in honor of Hooke which developed the Principia of the elasticity theory-was numerically simulated using the finite element method (FEM). The FEM is a numerical method used to perform structural analysis and is applicable to a large set of problems involving the solution of partial differential equations. Particularly, in the development of gravitational antennas the method has been used ${ }^{4,5}$ to model the modules used in the mechanical isolation system. To perform the numerical analysis, we used the NASTRAN software which determines the geometrical setup of nodes and elements, solves the dynamical equations corresponding to the structure and allows the visualization of the normal modes shapes.

\section{THE HOOKE APPARATUS: THE ISOLATION SYSTEM DESIGNED FOR NEWTON}

The main mechanical noise sources to be considered in gravitational detection are the background seismic and the human made cultural mechanical activities. In regions of low seismic activity the characteristic spectral behavior of the background scismic is known ${ }^{6}$ to follow the empirical expression $a(1 \mathrm{~Hz} / \nu)^{2} \mathrm{~m} /(\mathrm{Hz})^{1 / 2}$, where $\nu$ is the frequency and $a$ is a parameter that depends on the location at the Earth surface. In a seismically very quiet site $\left(a=10^{-8}\right)$, and supposing that the frequency of detection is about $2 \mathrm{KHz}$ the spectral density level of seismic noise will be $10^{-14} \mathrm{~m} / \mathrm{Hz}^{-1 / 2}$. This means that, if we hope to obtain sensitivity of about $10^{-22} \mathrm{~m} /(\mathrm{Hz})^{1 / 2}$, which is necessary to detect waves from the Virgo cluster of galaxies, then it is necessary to filter the seismic noise ${ }^{7}$ by a factor of $10^{-8}$, or $-160 \mathrm{~dB}$, at least. So it is necessary to construct an isolation system capable of providing this level of damping. The classic way to obtain such high damping factor is to use a series

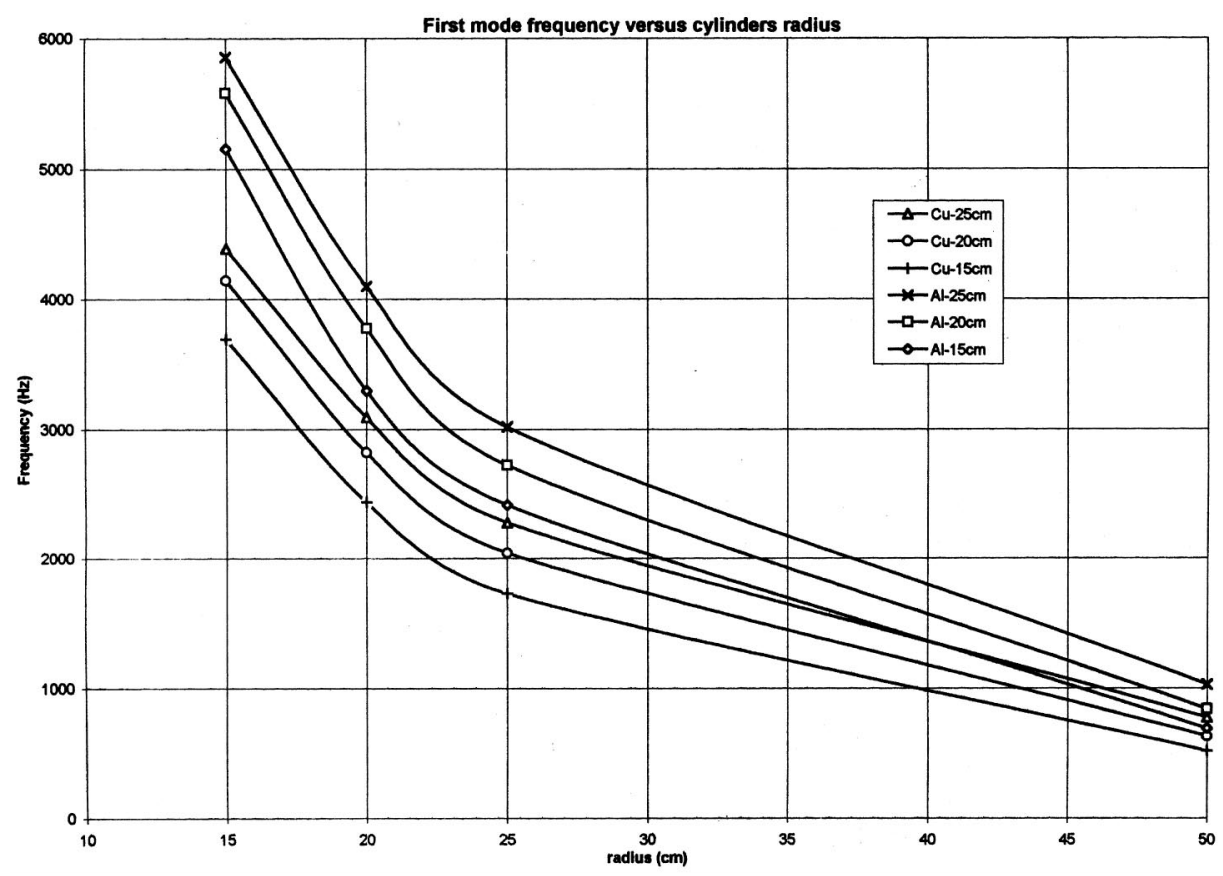

FIG. 2. The fundamental normal modes frequencies of cylinders of $\mathrm{Al}$ and $\mathrm{Cu}$ in function of the cylinders radius. Each curve corresponds to different heights $(15,20$, and $25 \mathrm{~cm})$. 


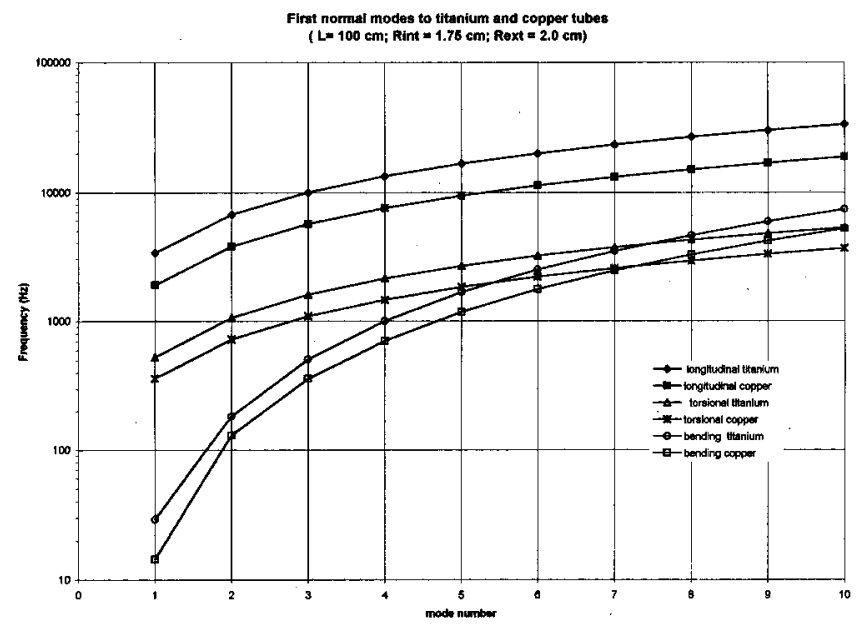

FIG. 3. The first 10 modes (bending, longitudinal and torsional resonances) of $1 \mathrm{~m}$ height copper and titanium tubes. The thickness is $0.25 \mathrm{~cm}$. From this configuration we can see that the first longitudinal mode and the fourth, fifth, sixth, and seventh bending or torsional modes are around $2000 \mathrm{~Hz}$, which is the detection range of interest.

of spring-mass modules in a multistage setup. As is well known, this type of structure works as a low-pass filter and so provides an attenuation to mechanical noises. This kind of isolation system is normally used both in interferometric ${ }^{8-10}$ antennas and in resonant ${ }^{11,12}$ antennas. As the resonant mass in the NEWTON antenna will be very high (about $5000 \mathrm{Kg}$ ) and since the isolation system will be inside the cryogenic chambers, we must use rigid springs ${ }^{13}$ (no air springs or

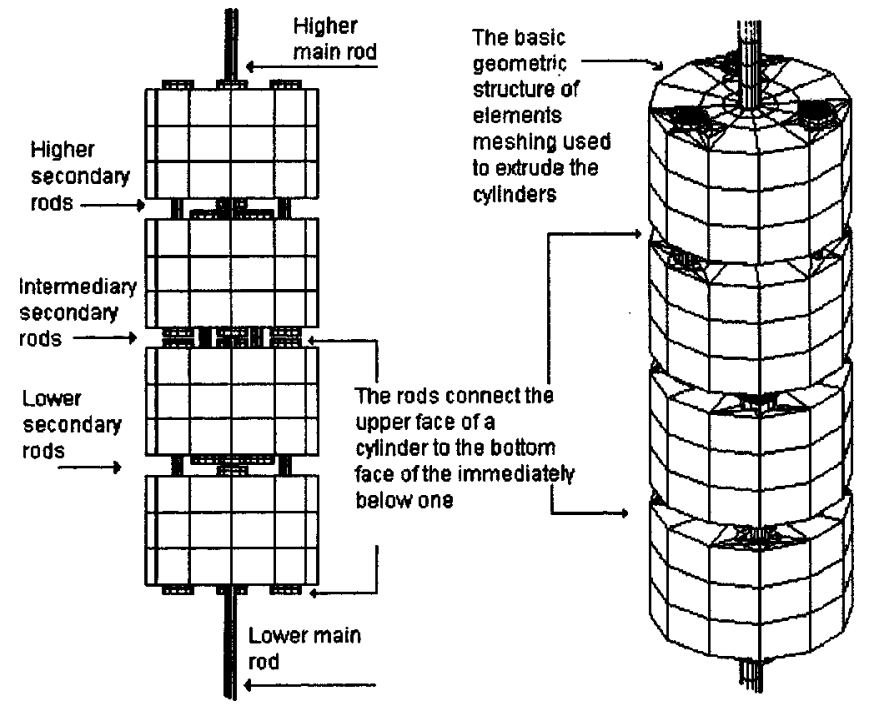

FIG. 5. Design and meshing of the HOOKE apparatus.

strings). Until now the simplest solution seems to be the use of hollow rods (or tubes) that connect large intermediate masses, forming a multistage coupled pendulum. Using hollow rods allows to easily realize the fine frequency tuning since there are more geometrical parameters to adjust.

\section{A. The Hooke apparatus geometric basic scheme}

In Fig. 1 we show schematics of the NEWTON antenna and its isolation system suspending the $100 \mathrm{~cm}$ diameter buckyball. In the figure, the dilution refrigerator, the three

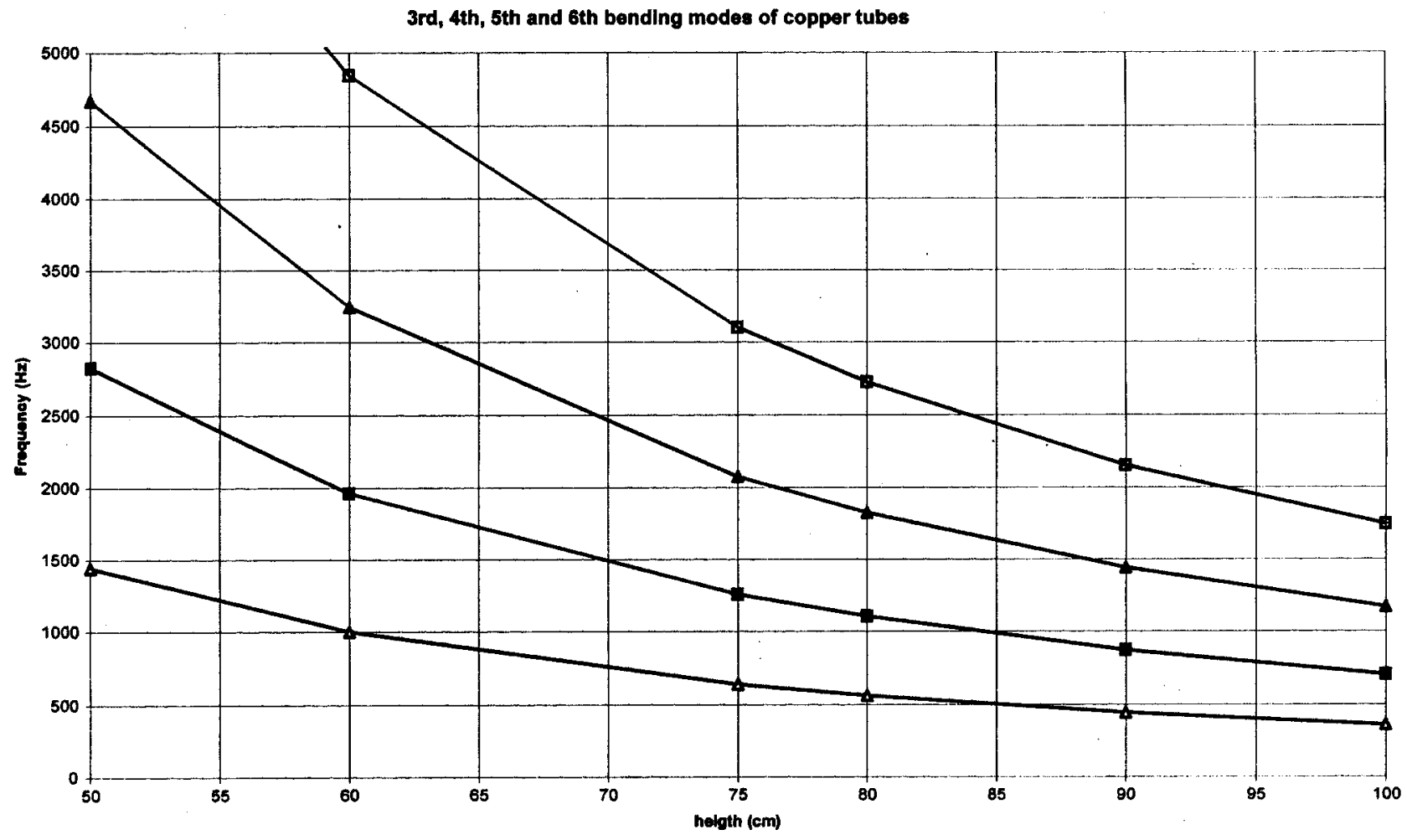

FIG. 4. Bending mode frequencies vs height for copper tubes with thickness of $0.25 \mathrm{~cm}$. The fourth, fifth, and sixth modes must be considered in the design of the isolation modules. 
TABLE I. The geometric configurations tested. The first column refers to the terminology defined in Fig. 5.

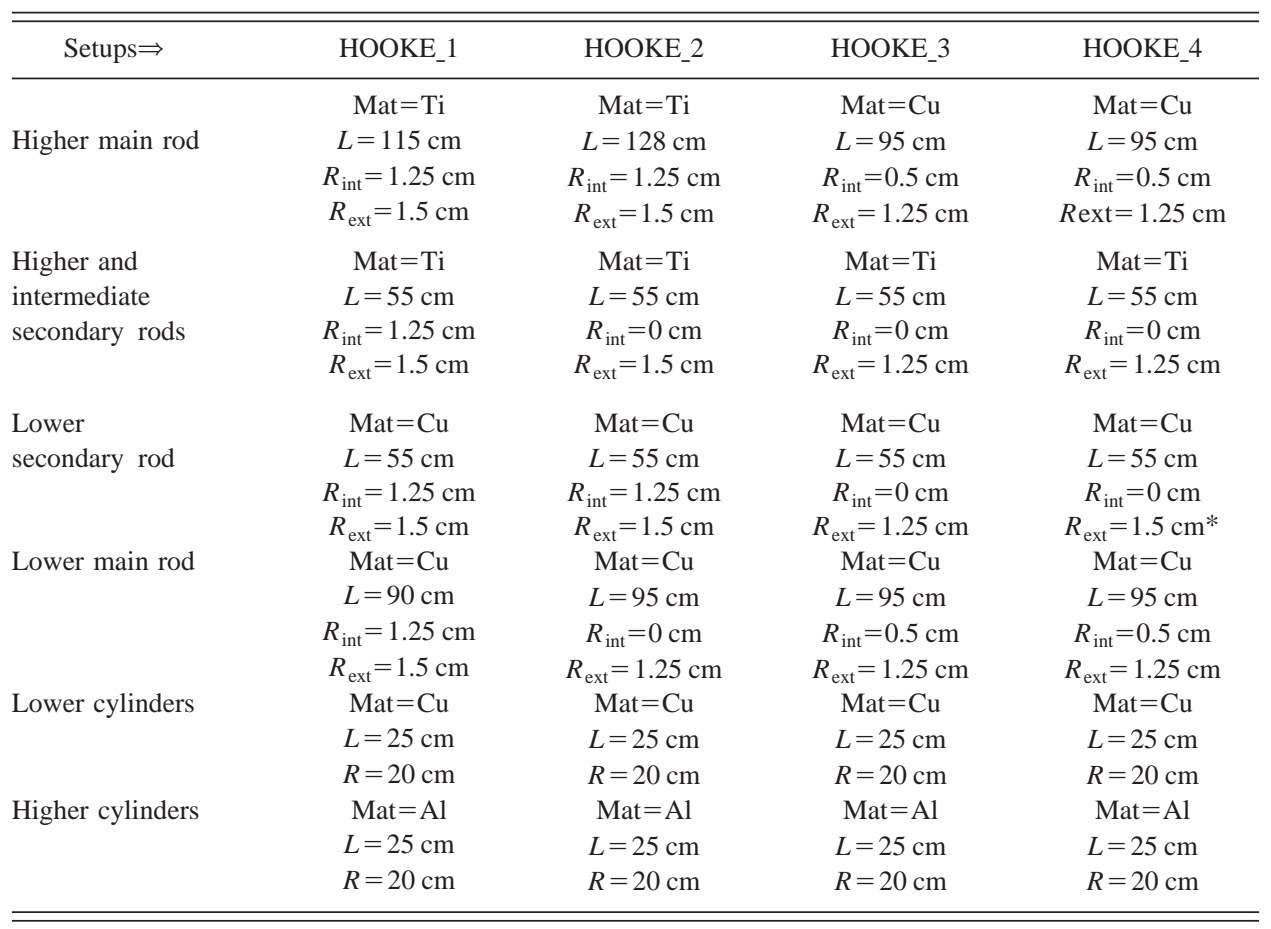

points support, the suspended buckyball and the HOOKE apparatus are represented.

The basic idea in the design is that the multiple stage pendulum is capable of reducing the mechanical vibrations in the spectral region of detection interest. A system with the cylinders joined together by hollow rods seems to be a good approach to obtain this. In our design we used four cylinders. Less than four cylinders could not be enough to obtain the necessary attenuation. More than four cylinders, of course, would result in a larger attenuation, but it would also have more internal modes which would make difficult the fine frequency tuning. Actually, the system will have some structural normal modes at low frequencies, which will provide mechanical damping at larger frequencies, but will also present a large number of internal resonances, corresponding to normal modes of each component of the structure (cylinders and hollow rods). Using four cylinders seemed to be a good accordance between these two factors.

So, the basic constraints involved in the design will be: (1) to use a cylinder small enough to have the first internal resonance at a frequency larger and far from the detection frequency; and (2) to choose the geometrical parameters of the hollow rod (internal and external radius and height) and so adjusting the elastic characteristics in a way to obtain a kind of spectral window, free of resonances, around the detection frequency.

\section{B. Normal modes and shapes of metallic cylinders}

We used the finite element method $^{14}$ to calculate the internal normal modes of metallic cylinders made of copper or aluminum. The normal modes frequencies depend, of course, on its geometric dimensions (diameter and height) and on the elastic characteristics of the material (copper or aluminum). The graph in Fig. 2 shows curves corresponding to the frequency of the fundamental normal mode (which corresponds to the lowest drum mode) in function of the cylinder radius and parametrized with the cylinder height, for both copper and aluminum.

In the curves we can easily note two regions with difference slopes, corresponding to the plate-disk behavior (when the diameter is larger than the height) and to the solid-prism behavior (as the height becomes equal to or larger than the diameter). Looking at the curves, we can choose the adequate cylinder dimensions that determine the first normal modes at frequencies as large as $3000 \mathrm{~Hz}$, which is far enough from the detection frequency. The chosen dimensions were: diameter of $40 \mathrm{~cm}$ and height of $25 \mathrm{~cm}$ for both aluminum and copper cylinders.

\section{Bending, axial, and torsional vibration of a metallic rod}

The elastic behavior of a hollow rod is well known. The main normal modes of interest are the bending (transverse vibrations) and the axial and torsional vibrations. The spectral distribution of these modes depends on its geometrical dimensions and elastic characteristics. In Fig. 3 are plotted the curves corresponding to the following equations ${ }^{15}$ for the first ten bending, longitudinal, and torsional modes of a rod with both the ends clamped:

$$
\begin{aligned}
& f_{i}=\left(\lambda_{i}^{2} / 2 \pi L^{2}\right)(E I / m)^{1 / 2} \quad \text { (bending modes); } \\
& f_{i}=\left(\lambda_{i} / 2 \pi L\right)(E / \mu)^{1 / 2} \quad \text { (longitudinal modes); } \\
& f_{i}=\left(\lambda_{i} / 2 \pi L\right)\left(C G / \mu I_{p}\right)^{1 / 2} \quad \text { (torsional modes), }
\end{aligned}
$$

where $\lambda_{i}=(2 i \pm 1) \times \pi / 2$ is a dimensionless parameter depending on the boundary conditions applied to the rod, $L$ is the length, $E$ is the Young modulus, $I$ is the area moment of inertia, $I_{p}$ is the torsion moment of inertia and $m$ is the mass 


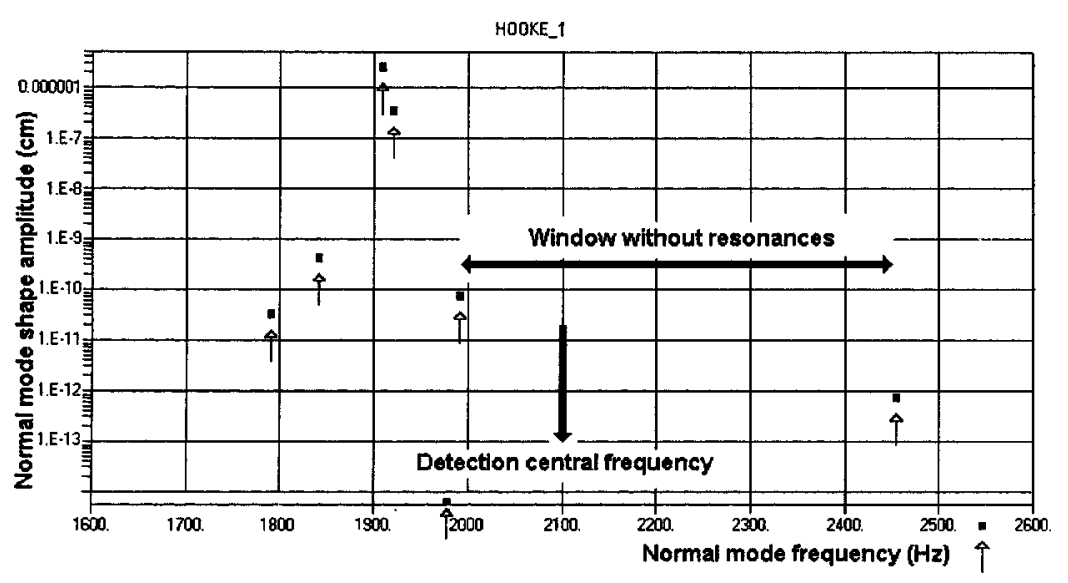

FIG. 6. Normal modes of HOOKE_1 setup.

per unit length of the rod, $\mu$ is the mass density, $C$ is the torsional constant of cross section, and $G$ is the shear modulus.

To design the vibrational isolation modules we need to consider the third, fourth, fifth, and sixth bending and torsional modes and the first and second longitudinal modes that are near to $2100 \mathrm{~Hz}$, since this frequency corresponds to the detection band of our antenna.

In Fig. 4 we have plotted the frequencies of the bending modes in function of the height of tubes. Using this figure it is possible to choose the best height for the isolation modules tubes. From the figure, it is easy to see that tubes $1 \mathrm{~m}$ long are bad because the sixth mode is very close to $2100 \mathrm{~Hz}$.

The same thing could be said for tubes of $85 \mathrm{~cm}$, since, in this case, the fifth mode will be at about $2100 \mathrm{~Hz}$. For example, two good possible heights for the tubes would be 95 and $80 \mathrm{~cm}$. It Is easy to see in the figure that tubes with these dimensions do not have modes in the neighborhood of $2100 \mathrm{~Hz}$. Similar plots can be made for the torsional and longitudinal modes allowing to adjust the tubes dimensions in way to set the internal resonances far from the detection frequency.

\section{THE FINITE ELEMENT ANALYSIS: NUMERICAL SIMULATIONS (NORMAL MODES)}

Using the above considerations we defined the first geometric setup for the vibrational isolation system. We have numerically modeled the structure using a large number of elements (about 10000 , which is a pratical limit to our computer system) to increase the FEM calculation accuracy and a geometrical configuration as symetrical as possible (see Fig. 5) to simplify the numerical solutions. In Table I the columns correspond to the four models we have designed and analyzed using the FEM. The HOOKE_1 was the first setup we designed considering the dynamical constraints we show above (Sec. II C). As a first step of the analysis we used the FEM to calculate the stress of the structure, considering the total weight (including, of course, the buckyball mass). The results shown that the stress in each element of the structure was about one order of magnitude smaller than the yield strength for the materials.

The other models were designed and analyzed in an iterative way, changing the mechanical parameters (external and internal radius of the hollow rods etc.) in order to move the resonances out of the spectral region of detection interest. The HOOKE_4 is the setup we obtained after four design iterations.

In the Fig. 6 we show the calculated normal modes, using the finite element method, for the first setup (the HOOKE_1 apparatus) in a spectral band around the detector characteristic frequency. With this first setup we obtained a kind of spectral window, from 1990 to $2450 \mathrm{~Hz}$, where the HOOKE structure has no internal resonances. However, this spectral window is relatively small and not symmetric with respect to the characteristic frequency.

So we changed the geometric setup trying to increase the

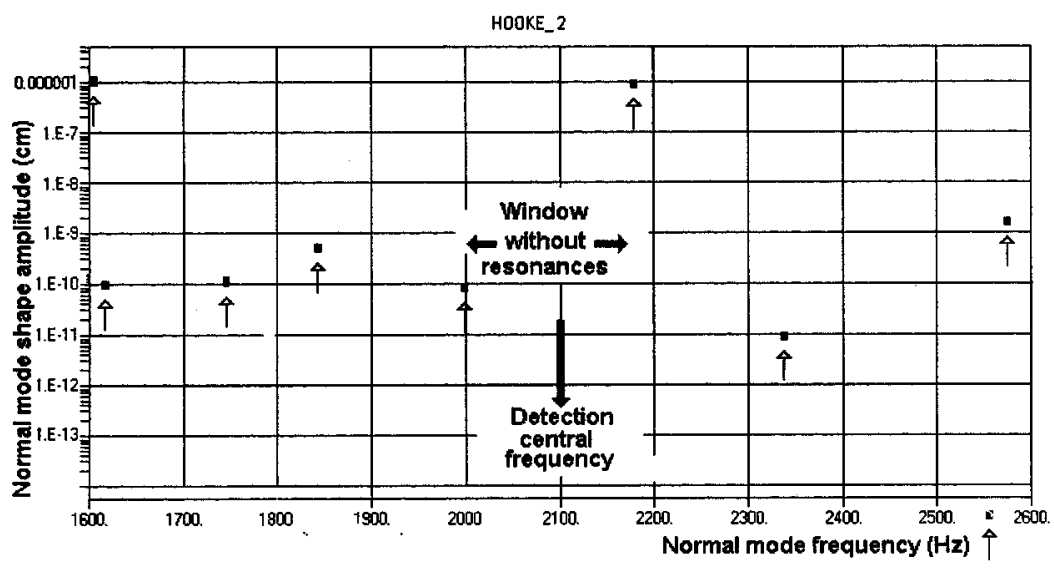

FIG. 7. Normal modes of HOOKE_2 setup. 


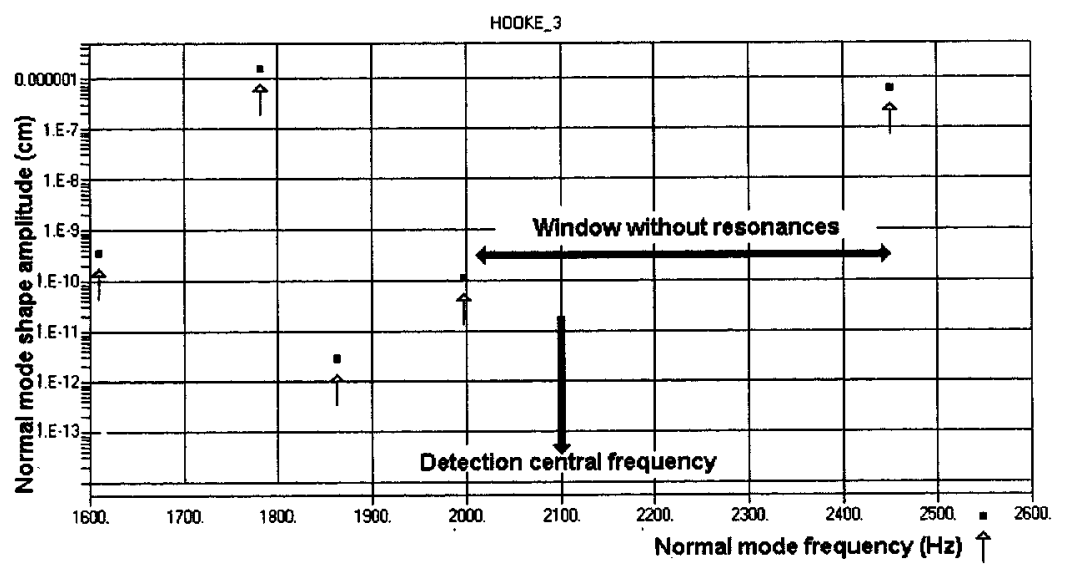

FIG. 8. Normal modes of HOOKE_3 setup.

spectral window. We first identified the normal modes at the frequencies of 1789, 1841, 1909, 1920, 1978, 1991, and $2455 \mathrm{~Hz}$, respectively as: (a) the torsional mode of the lowermain rod, (b) the bending modes of the lower-secondary rods, (c) the longitudinal mode of the lower-main rod, (d) the bending mode of the lower-main rod, (e) the bending mode of the higher-main rod, (f) the transversal modes of the lower-secondary rods, and $(\mathrm{g})$ the bending mode of the higher-secondary rods.

So we have made some small changes in the corresponding geometric parameters of the isolation system, in order to move some of those resonances out of the interest spectral range: (a) a longer but thinner lower-main rod would have the bending modes at higher $(1920 \mathrm{~Hz} \rightarrow)$ and the torsional and longitudinal modes at a lower $(\leftarrow 1789 \mathrm{~Hz}, \leftarrow 1909 \mathrm{~Hz})$ frequencies; (b) a longer higher-main hollow rod would have the bending modes at a lower frequency $(\leftarrow 1978 \mathrm{~Hz})$; and (c) the intermediate-secondary titanium rods would have the transversal normal resonances at higher frequencies (2445 $\mathrm{Hz} \rightarrow$ ) with respect to the tubes. In this first iteration we have made no changes in the lower-secondary rods. The corresponding modes $(1841$ and $1991 \mathrm{~Hz}$ ) will be moved only in the third step.

These changes produced the HOOKE_2 setup (see Table I). We calculated the normal modes for this configuration and the results are shown in Fig. 7. In the figure we can see that with the HOOKE_2 setup the mode corresponding to the bending of the higher main rod $(1978 \mathrm{~Hz}$ in HOOKE_1) "disappeared" (in truth it goes outside the spectral range we are considering) but, however, another harmonic of this resonance "appears" at $2338 \mathrm{~Hz}$. Unfortunately, also the resonance corresponding to the bending of the lower main rod (1920 Hz in HOOKE_1) moved less than we were hoping and appears at $2178 \mathrm{~Hz}$, showing that the adjustment made at this element of the structure was insufficient. In order to easily obtain the best setup, we calculated the normal modes of isolated copper tubes and rods with different dimensions. The calculations suggested the dimensions used in the HOOKE_3. Of course this is not the best configuration since a copper higher-main rod could reintroduce some heat from the environment to the cooled buckball. However, as we are just testing the design methodology and since the height of the higher rod is a free parameter of our project at this step, we chose to impose that the main higher and lower rods be equal in order to simplify the calculations. With this setup we have calculated the normal modes. Figure 8 shows these results.

It is easy to see in Fig. 8 that with these changes, we obtained a large spectral window, from $1860 \mathrm{~Hz}$ to $2449 \mathrm{~Hz}$, where only a resonance $(1997 \mathrm{~Hz})$ exists.

This resonance corresponds to transversal vibrations of the lower-secondary rods. Trying to reduce the frequency of this resonance we made the rod a bit larger in its middle region, as is shown in Fig. 9, increasing the mass of the rod without changing the stiffness. This small change defined the HOOKE_4 and the calculated normal modes of this setup is shown in Fig. 10. With this setup we were able to move the resonance from 1997 to $1828 \mathrm{~Hz}$ resulting in a $559 \mathrm{~Hz}$ large spectral window free of resonances $(1861 \rightarrow 2420 \mathrm{~Hz})$.

Once we obtained this spectral window without resonances, we concluded that the design methodology was good enough for our proposes. Adjusting the geometric parameters of the structure, it is possible to arrange the resonances in the way we want, as far as necessary (some hundreds of $\mathrm{Hz}$ ) around a desired frequency. At this stage we use the FEM again to calculate the normal modes from 0 to $3000 \mathrm{~Hz}$ in order to determine the dynamical behavior of the structure and to simulate its transfer function (Fig. 11).

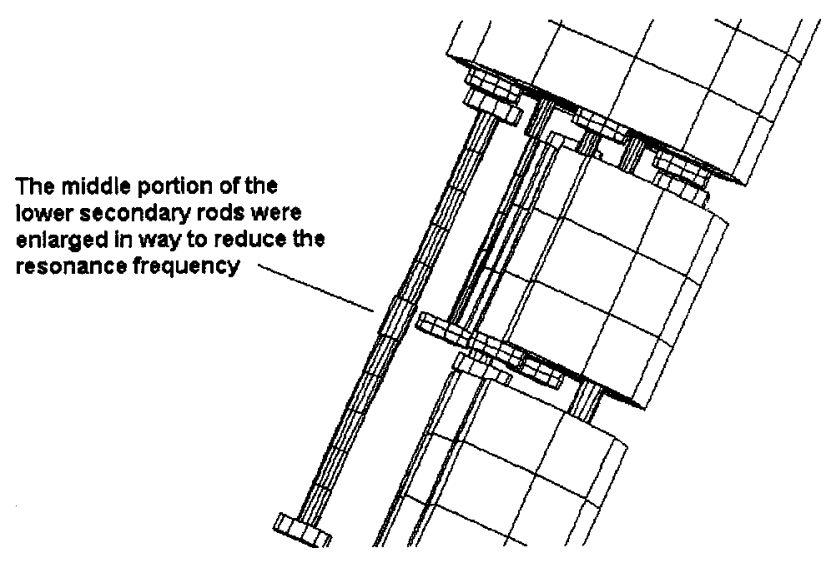

FIG. 9. Details of the middle ring appended to the lower secondary rod. 


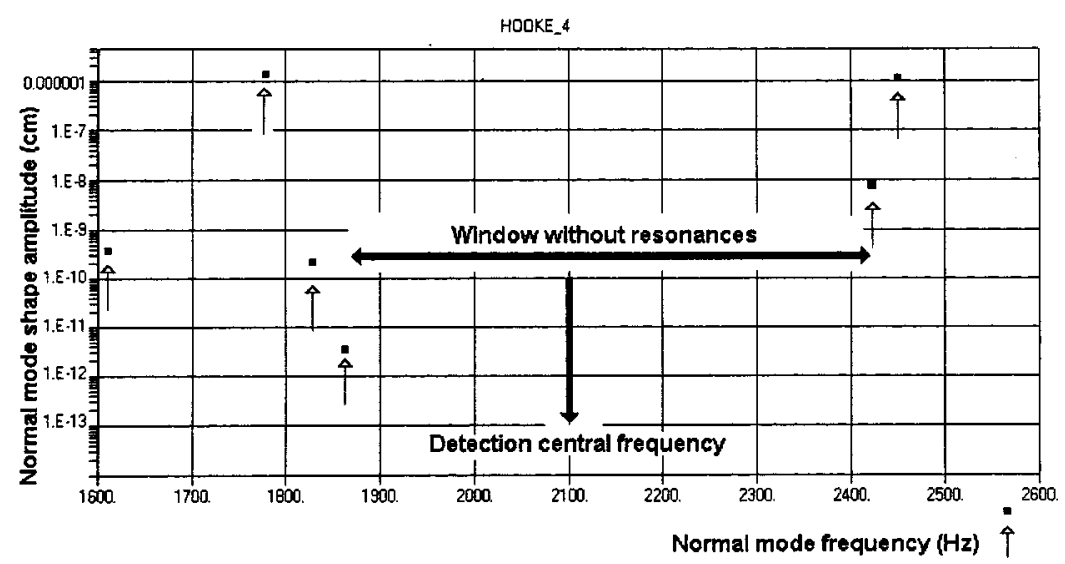

FIG. 10. Normal modes of HOOKE_4 setup.

\section{DISCUSSION: THE HOOKE_4 FREQUENCY RESPONSE CURVES}

The frequency response of a mechanical structure depends, of course, on all its normal modes, however, are mainly the low frequency structural modes that determine the system damping characteristics. So, even if the HOOKE structure is not directly comparable with a simple multistage pendulum, since we must to consider also the internal high frequency resonances of the rigid springs, we can hope to achieve a good attenuation level at frequencies far enough from the first modes. To calculate the frequency response we need to solve the dynamical equations of the structure, considering a force of excitation applied to the structure. The equations could be written in the matrix form ${ }^{16}$

$$
[M]\{\ddot{x}(t)\}+[B]\{\dot{x}(t)\}+[K]\{x(t)\}=\{P(w)\} e^{i w t},
$$

where $[M],[B]$, and $[K]$, the mass, damping, and stiffness matrices, contains the mechanical characteristics of each element of the structure and $\{x(t)\}$ is the displacements vector.

The solution, for harmonic excitation, if we do not consider the viscous damping, could be

$$
\left[-w^{2} M+K\right]\{x(w)\}=\{P(w)\}
$$

and the frequency response will be calculated by

$$
\frac{\{x(w)\}}{\{P(w)\}}=\frac{1}{\left[-w^{2} M+K\right]} .
$$

It would be necessary to do the calculation considering frequencies from 0 to $3000 \mathrm{~Hz}$, which means computing all amplitudes in the six degrees-of-freedom for each element of the HOOKE apparatus for each frequency step. Unfortunately, since our model has more than 10000 elements and more than 14000 nodes, this task is greater than our computer set would be capable of performing.

So, it was necessary to use the modal approach to compute the frequency response. The modal method makes the numerical solution more efficient since it uncouples the equations and so reduces their size. This method uses the modal shapes to transform the problem, describing the system in terms of the modes behavior. The first step is to relate the physical coordinates $\{x(w)\}$ to the modal coordinates $\{\xi(w)\}$ using:

$$
\{x\}=[\phi]\{\xi(w)\} e^{i w t},
$$

where $[\phi]$ are the mode shapes.

With these relations, the equations of motion can be written as a set of uncoupled systems

$$
-w^{2} m_{i} \xi_{i}(w)+k_{i} \xi_{i}(w)=p_{i}(w),
$$

where $p_{i}$ is the $i$ th modal force, $m_{i}$ is the $i$ th modal mass, and $k_{i}$ is the $i$ th modal stiffness. The equation represents a exact solution if all the modes are used. Since, in general, only the first modes are used, the solution represents an approximation. However, we used all the modes from 0 to $2500 \mathrm{~Hz}$ to solve our problem, and so the solution represents a very good approximation to the system dynamics at the frequency of $2100 \mathrm{~Hz}$. In our calculations we have used a forcing vector $p_{i}(w)$ which was defined as

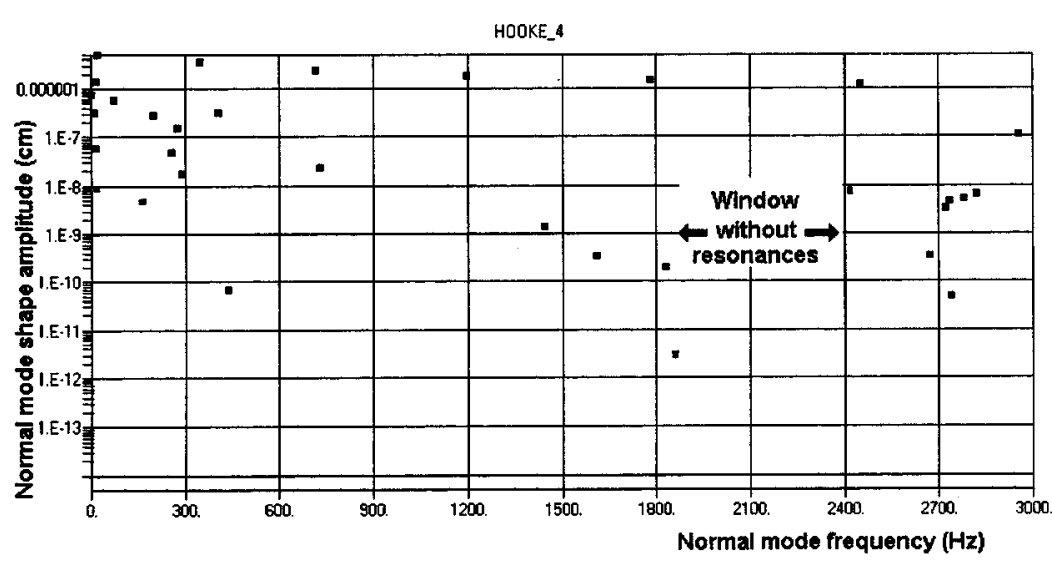

FIG. 11. The normal modes frequencies of HOOKE_4 from 0 to $3000 \mathrm{~Hz}$. 
HOOKE-4 Frequency Response

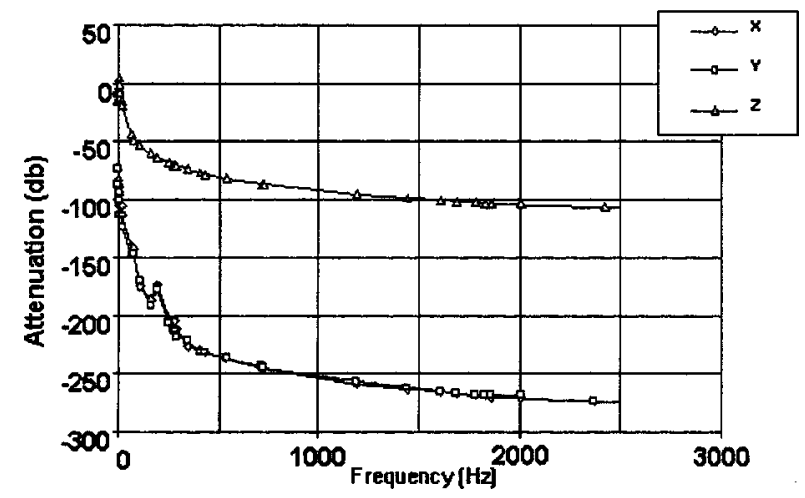

FIG. 12. The $X, Y$, and $Z$ frequency response of HOOKE_4 from 0 to $3000 \mathrm{~Hz}$.

$$
p_{i}(w)=\sum_{i=1}^{n} f_{i} \times g(w)
$$

where $f_{i}$ is a constant force and $g(w)=\alpha w^{-2}$ is a dimensionless weight function which gives the spectral dependence corresponding to the seismic noise.

The calculations, based on these normal modes, resulted in the $X, Y$, and $Z$ frequency response curves, shown in Fig. 12 , corresponding to a point in the lower end of HOOKE apparatus (which is attached to the baricenter of the buckball). We can see that, in both $X$ and $Y$ cases, the attenuation at $2100 \mathrm{~Hz}$ is about $-250 \mathrm{~dB}$, which is large enough for our proposals. However, in the $Z$ case, the attenuation level is only about $-100 \mathrm{~dB}$ which is reasonable if we consider that the structure is much more rigid to movements in the $Z$ direction than in the other ones. So, to obtain a larger attenuation factor in the $Z$ direction, it will be necessary to put another extra-isolation module in series with the HOOKE apparatus. A possibility is to use vertical air springs outside the cryogenic chambers. This kind of isolation modules are commonly used in gravitational waves experiments and can give a very low resonance frequency which means a large Z-attenuation factor at $2100 \mathrm{~Hz}$.

The methodology we have proposed permits easily design isolation systems for resonant mass up to some thousands of $\mathrm{Kg}$. It is relatively easy to adjust the size and shape of the intermediate masses and the dimensions of the hollow rods to open a window free from resonances that includes the detector bandwidth. Of course the precise spectral behavior of the mechanism will depend on the final dimensions of the structure and also on the mounting details of the structure. In spite of this, and based on our simulations, we believe that using an "HOOKE-like" system it would be possible to achieve attenuation factors large enough to be used in medium sized resonant gravitational radiation antennas. If we consider that the mechanical noise level at the detection frequency is about $10^{-14} \mathrm{~m} \mathrm{~Hz}^{1 / 2}$ then the level of attenuation we obtained with the HOOKE apparatus will result in a noise level of $10^{-24} \mathrm{~m} \mathrm{~Hz}^{1 / 2}$, which would mean that, the detector noise would be dominated by the thermal noise.

\section{ACKNOWLEDGMENTS}

This work has been funded by grants from FAPESP (Proc. Nos. 1996/2238-7 and 97/14437-7) and CNPq [Proc. No. 300 746/95-4 (NV) and No. 300619/92-8].

${ }^{1}$ R. A. Hulse and J. H. Taylor, Astrophys. J., Lett. Ed. 195, L51 (1975).

${ }^{2}$ For an update on detectors AURIGA, NAUTILUS, NIOBE, EINSTEIN, TIGA, ELSA, and GRAIL, see the Proceedings of the 2nd E. Amaldi Conference, Gravitational Waves edited by E. Coccia, G. Pizzela, and G. Veneziano (World Scientific, Singapore, 1998); Proceedings of the OMNI I Workshop, Omnindirectional Gravitational Radiation Observatory edited by W. F. Velloso Jr., O. D. Aguiar, and N. S. Magalhães (World Scientific, Singapore, 1997).

${ }^{3}$ SCHENBERG-We choose the name of our detector in honor of one of the most important Brazilian physicist. Mário Schenberg was born in Recife in 1914, and died in São Paulo in 1990. He published more than 100 papers in theoretical and experimental physics, astrophysics, statistical and quantum mechanics, relativity, quantum field theory, and wrote many papers in mathematics. In particular, he developed theoretical studies about the nuclear physics process occuring in the interior of supernovas, which he named "Urca process," in collaboration with G. Gamow.

${ }^{4}$ C. A. Cantley, J. Hough, N. A. Robertson, and A. Greenhalgh, Rev. Sci. Instrum. 63, 4 (1992).

${ }^{5}$ T. L. Aldcroft, P. F. Michelson, R. C. Taber, and F. A. McLoughlin, Rev. Sci. Instrum. 63, 8 (1992).

${ }^{6}$ A. Araya, K. Kawabe, T. Sato, N. Mio, and K. Tsubono, Rev. Sci. Instrum. 64, 5 (1993).

${ }^{7}$ D. G. Blair, D. E. McClelland, H. A. Bachor, and R. J. Sandeman, in The Detection of Gravitational Waves, edited by D. G. Blair (Cambridge University Press, Cambridge, 1991), p. 43.

${ }^{8}$ R. Delfabro, A. Di Virgilo, A. Giazotto, H. Kautzky, V. Montelatici, and D. Passuelo, Phys. Lett. A 124, 253 (1989).

${ }^{9}$ L. Difiore et al., in Proceedings of the First International Workshop for an Omnidirectional Gravitational Radiation Observatory, edited by W. F. Velloso, Jr., O. D. Aguiar, and N. S. Magalhães (World Scientific, Singapore, 1997), p. 241.

${ }^{10} \mathrm{~B}$. Barish, in Proceedings of the First International Workshop for an Omnidirectional Gravitational Radiation Observatory, edited by W. F. Velloso, Jr., O. D. Aguiar, and N. S. Magalhães (World Scientific, Singapore, 1997), p. 260.

${ }^{11} \mathrm{P}$. Astone et al., in Proceedings of the First International Workshop for an Omnidirectional Gravitational Radiation Observatory, edited by W. F. Velloso, Jr., O. D. Aguiar, and N. S. Magalhães (World Scientific, Singapore, 1997), p. 39.

${ }^{12}$ D. G. Blair, I. S. Heng, E. N. Ivanov, M. E. Tobar, P. J. Turner, and F. J. van Kann, in Proceedings of the First International Workshop for an Omnidirectional Gravitational Radiation Observatory, edited by W. F. Velloso, Jr., O. D. Aguiar, and N. S. Magalhães (World Scientific, Singapore, 1997), p. 27.

${ }^{13}$ S. M. Merkowitz, E. Coccia, V. Fafone, G. Raffone, M. Schipilliti, and M. Visco, Rev. Sci. Instrum. 70, 2 (1999).

${ }^{14}$ R. J. Astley, in Finite Elements in Solids and Structures (Chapman and Hall, London, 1992).

${ }^{15}$ R. D. Belvins, in Formulas for Natural Frequency and Mode Shape (Krieger, Florida, 1995)

${ }^{16}$ M. N. BismarcK-Nasr, in Finite Elements in Applied Mechanics, São Paulo, 1993 (unpublished). 